\title{
Penumbral thermal structure below the visible surface
}

\author{
J. M. Borrero ${ }^{1}$, M. Franz ${ }^{1}$, R. Schlichenmaier ${ }^{1}$, M. Collados ${ }^{2,3}$, and A. Asensio Ramos ${ }^{2,3}$ \\ 1 Kiepenheuer-Institut für Sonnenphysik, Schöneckstr. 6, 79104 Freiburg, Germany \\ e-mail: borrero@leibniz-kis.de \\ 2 Instituto de Astrofísica de Canarias, Avd. Vía Láctea s/n, 38205 La Laguna, Tenerife, Spain \\ 3 Departamento de Astrofísica, Universidad de La Laguna, 38205 La Laguna, Tenerife, Spain
}

Recieved 9 March 2017 / Accepted 28 April 2017

\begin{abstract}
Context. The thermal structure of the penumbra below its visible surface (i.e., $\tau_{5} \geq 1$ ) has important implications for our present understanding of sunspots and their penumbrae: their brightness and energy transport, mode conversion of magneto-acoustic waves, sunspot seismology, and so forth.

Aims. We aim at determining the thermal stratification in the layers immediately beneath the visible surface of the penumbra: $\tau_{5} \in$ $[1,3]$ ( $\approx 70-80 \mathrm{~km}$ below the visible continuum-forming layer)

Methods. We analyzed spectropolarimetric data (i.e., Stokes profiles) in three Fe I lines located at $1565 \mathrm{~nm}$ observed with the GRIS instrument attached to the $1.5-\mathrm{m}$ solar telescope GREGOR. The data are corrected for the smearing effects of wide-angle scattered light and then subjected to an inversion code for the radiative transfer equation in order to retrieve, among others, the temperature as a function of optical depth $T\left(\tau_{5}\right)$.

Results. We find that the temperature gradient below the visible surface of the penumbra is smaller than in the quiet Sun. This implies that in the region $\tau_{5} \geq 1$ the penumbral temperature diverges from that of the quiet Sun. The same result is obtained when focusing only on the thermal structure below the surface of bright penumbral filaments.

Conclusions. We interpret these results as evidence of a thick penumbra, whereby the magnetopause is not located near its visible surface. In addition, we find that the temperature gradient in bright penumbral filaments is lower than in granules. This can be explained in terms of the limited expansion of a hot upflow inside a penumbral filament relative to a granular upflow, as magnetic pressure and tension forces from the surrounding penumbral magnetic field hinder an expansion like this.
\end{abstract}

Key words. sunspots - Sun: magnetic fields - Sun: infrared - Sun: photosphere

\section{Introduction}

The thermal structure of sunspots, in particular below the visible surface, is of great importance for a number of scientific studies. It determines the sound speed and therefore the inference of the subsurface structure of sunspots through the calculation of travel times through local helioseismology (see Couvidat et al. 2006; Moradi et al. 2010; Gizon et al. 2009; Khomenko \& Collados 2015, and references therein). It also establishes the location of the $\beta=1$-surface (i.e., the region where magnetic and gas pressure are equal) where most of the mode conversion of magnetoacoustic waves takes place (Cally 2005; Schunker \& Cally 2006; Khomenko \& Cally 2012). Furthermore, a proper knowledge of the thermal stratification can also be employed to assess the realism of three-dimensional magnetohydrodynamic simulations of sunspots (Heinemann et al. 2007; Rempel et al. 2009; Rempel 2012). It also imposes strong observational constraints on the different models that aim at explaining the onset of convection and heat transport in sunspot penumbrae: convective rolls (Danielson 1961), flows along magnetic flux tubes (Schlichenmaier et al. 1999), or overturning convection in fieldfree gaps (Spruit \& Scharmer 2006; Scharmer \& Spruit 2006). Finally, it can help elucidate whether the subsurface structure of the penumbra is better explained in terms of the monolithic (Deinzer 1965; Meyer et al. 1974) or cluster/spaghetti (Parker 1979) models.

Unfortunately, the subsurface layers are not easily accessible. For instance, the analysis of spectral lines and their polarization signals (i.e., spectropolarimetry) allows inferring the physical properties of solar and stellar atmospheres (Chandrasekhar 1960; del Toro Iniesta 2003) as a function of the continuum optical depth $\tau_{\mathrm{c}}$, where $\tau_{\mathrm{c}}=1$ is considered the deepest observable layer and is oftentimes referred to as the continuum-forming layer. However, the wavelength dependence of the continuum optical depth offers the possibility of observing slightly deeper or higher layers of the solar atmosphere by observing different wavelengths (Chandrasekhar \& Breen 1946). The observed surface of the Sun (see Fig. 9 in Borrero et al. 2016) is located about $70-80 \mathrm{~km}$ deeper at $1565 \mathrm{~nm}$ (nearinfrared) than at $630 \mathrm{~nm}$ (visible): $z\left(\tau_{15}=1\right)-z\left(\tau_{6}=1\right) \approx$ $70-80 \mathrm{~km}$. Here, the subindexes 15 and 6 indicate wavelengths of $1565 \mathrm{~nm}$ and $630 \mathrm{~nm}$, respectively. If instead of using two different reference wavelengths we employ only the commonly used reference wavelength of $500 \mathrm{~nm}$, it can be shown (see Fig. 10 in Borrero et al. 2016) that the response function to the temperature for spectral lines at $630 \mathrm{~nm}$ peaks at $\tau_{5} \approx 1$, while for spectral lines at $1565 \mathrm{~nm}$, it peaks at around $\tau_{5} \approx 3$. In this work we exploit this property to investigate the penumbral thermal stratification $T\left(\tau_{5}\right)$ in the optical depth range $\tau_{5} \in[1,3]$, that is, in the first $\approx 70-80 \mathrm{~km}$ below its visible surface.

\section{Observations and inversion results}

The observations employed in this work correspond to spectropolarimetric data (i.e., the Stokes vector as a function of 

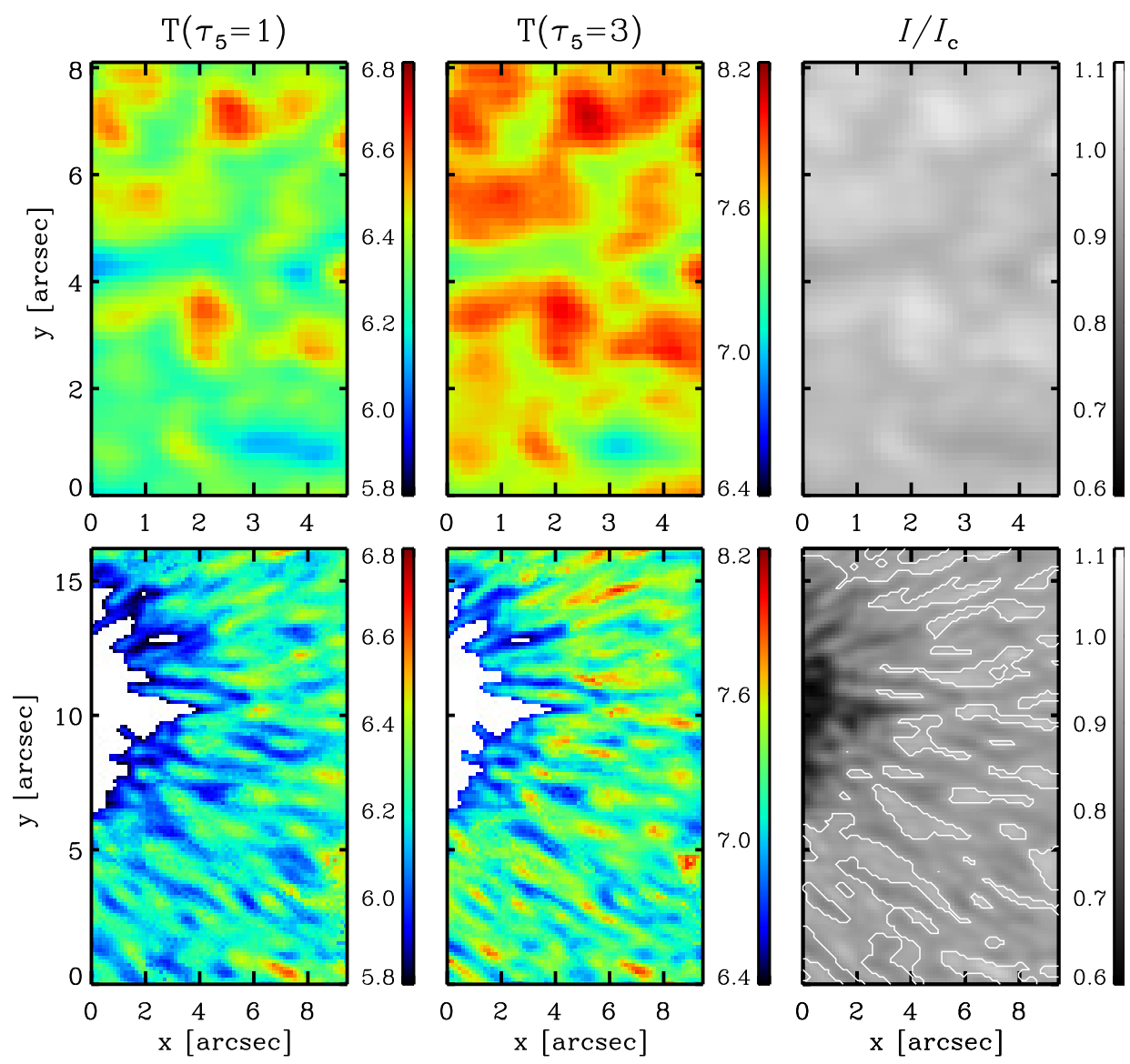

Fig. 1. Temperatures in two different regions: quiet Sun (top) and penumbra (bottom). Results at optical depths $\tau_{5}=1$ and $\tau_{5}=3$ are displayed in the left and middle panels, respectively. Right panels show the normalized continuum intensity images. White contours in the bottom right panel (continuum intensity in the penumbra) enclose bright penumbral filaments, whereas white regions in the penumbral temperature maps (left and middle bottom panels) indicate the umbra. All values of the temperature are given in thousands of Kelvin.

wavelength $I(\lambda)=(I, Q, U, V))$ in three Fe I lines at $1565 \mathrm{~nm}$. Although the spectral region contains six neutral iron spectral lines with a spectral resolution of $40 \mathrm{~m}^{\circ}$ pix $^{-1}$, here we analyze only the three with the largest Landé factor and cleanest continuum: Fe I $1564.8 \mathrm{~nm}$, Fe I $1565.2 \mathrm{~nm}$, and Fe I $1566.2 \mathrm{~nm}$. The atomic parameters of these spectral lines can be found in Table 1 in Borrero et al. (2016). The data were acquired with the GREGOR Infrared Spectrograph (GRIS; Collados et al. 2012) coupled with the Tenerife Infrared Polarimeter (TIP2; Collados 2007) that is attached to the 1.5-m GREGOR telescope (Schmidt et al. 2012). The target, NOAA 12049, was observed on May 3, 2014, very close to disk center (heliocentric angle $\Theta=6.5^{\circ}$ ). All details of the data reduction and postprocessing can be found in Borrero et al. (2016), Franz et al. (2016), Lagg et al. (2016), Martínez González et al. (2016). In addition, a PCA-based deconvolution (principal component analysis; see Ruiz Cobo \& Asensio Ramos 2013) was performed in order to account for an estimated $20 \%$ of wide-angle scattered light in the observations. The noise in the final data is about $10^{-3}$ (in units of the quiet-Sun continuum intensity), and the spatial resolution is estimated to be about $0.4-0.45^{\prime \prime}$.

While the size of the scanned region is $53 \times 45 \operatorname{arcsec}^{2}$ (see bottom panel in Fig. 1 in Borrero et al. 2016), in this work we focus on two distinct smaller regions. The first region is a quietSun patch with an area of $4.9 \times 8.3 \operatorname{arcsec}^{2}$ that contains about 2200 pixels. The second region is a $9.6 \times 16.5 \operatorname{arcsec}^{2}$ penumbral patch located on the limb side that contains about 8600 pixels. The right panels in Fig. 1 display the normalized continuum intensity in these regions. The normalization is done such that the average continuum intensity in the quiet-Sun region is unity.
The PCA-deconvolved spectropolarimetric data have been analyzed with the SIR (Ruiz Cobo \& del Toro Iniesta 1992) inversion code in order to retrieve the physical parameters (temperature, magnetic field, line-of-sight velocity, etc.) as a function of optical depth $\tau_{5}$. Owing to the removal of scattered light, we consider only one atmospheric component within each pixel (Borrero et al. 2016). In order to minimize the probability of arriving at a local minimum, the inversion at each pixel has been repeated ten times employing randomly generated initial guess models. These initial models feature $\tau$-independent values (randomly generated using a uniform distribution of values) in the kinematic and magnetic parameters. The thermal stratification is generated by performing random perturbations (also using a uniform distribution) at different optical depth locations on the HSRA model (Gingerich et al. 1971) and reinterpolating $T\left(\tau_{5}\right)$ in between these locations. Out of the ten inversion results we kept only the solution with the smaller $\chi^{2}$. Because in this work we are mostly interested in the temperature stratification $T\left(\tau_{5}\right)$, which is mostly encoded in Stokes $I$, we gave all four Stokes parameters equal weight in the inversion $w_{i, q, u, v}=1$ (cf. Borrero et al. 2016). During the inversion, the temperature was allowed to change in three nodes, while the components of the magnetic field $(B, \gamma, \phi)$ and the line-of-sight velocity $v_{\operatorname{los}}$ were allowed to change in two nodes when inverting the penumbral area, but only one node in the quiet-Sun area.

Figure 1 shows the resulting temperatures at two optical depths $\tau_{5}=1$ (left) and $\tau_{5}=3$ (middle) in the quiet-Sun (upper panels) and penumbral regions (bottom panels). The temperatures clearly closely follow the continuum intensity maps in the right panels of the same figure. For visualization purposes, the umbral region, defined as those regions where the 
Table 1. Mean temperatures (in Kelvin) at two different optical depths and their difference.

\begin{tabular}{cccc}
\hline \hline Region & $\bar{T}\left(\tau_{5}=1\right)$ & $\bar{T}\left(\tau_{5}=3\right)$ & $\Delta \bar{T}$ \\
\hline Quiet Sun & $6338[ \pm 67]$ & $7628[ \pm 80]$ & $1290[ \pm 104]$ \\
Granules & $6412[ \pm 67]$ & $7756[ \pm 80]$ & $1344[ \pm 104]$ \\
Intergranules & $6266[ \pm 67]$ & $7502[ \pm 80]$ & $1236[ \pm 104]$ \\
Penumbra & $5964[ \pm 67]$ & $6927[ \pm 80]$ & $963[ \pm 104]$ \\
Bright filaments & $6081[ \pm 67]$ & $7138[ \pm 80]$ & $1057[ \pm 104]$ \\
\hline
\end{tabular}

Notes. Errors have been estimated through a Monte Carlo-like simulation of Stokes profiles synthesized from three-dimensional magnetohydrodynamic simulations (Rempel 2011, 2012) that were inverted in the same fashion as described in Sect. 2 after adding photon noise to a level of $10^{-3}$.

continuum intensity is lower than 0.85 , is drawn in white on the temperature maps. Regions where the temperature is below the lower limit of the color bars are also shown in white. On the other hand, white contours in the continuum intensity map of the penumbra (bottom right panel) indicate the location of bright penumbral filaments. Pixels belonging to bright filaments are selected as those penumbral pixels where the continuum intensity is above the mean continuum intensity at the same radial distance from the sunspot center as the pixel subjected to scrutiny. As expected, the temperature in these bright filaments is generally higher than in the dark penumbral regions. Table 1 summarizes the mean temperatures at optical depths $\tau_{5}=1$ and 3, as well as their difference, in different regions: average quiet Sun, granules only, intergranules only, average penumbra, and bright penumbral filaments.

\section{Discussion and interpretation}

\subsection{Penumbra and bright filaments vs. average quiet Sun}

A particularly interesting feature is the finding that the difference of the mean temperatures between the quiet Sun and the penumbra is larger at $\tau_{5}=3$ than at $\tau_{5}=1$ (see Table 1 ). At $\tau_{5}=1$, the average quiet Sun is about $(374 \pm 95) \mathrm{K}$ hotter than the average penumbra, while this difference increases to $(701 \pm 113$ $\mathrm{K}$ at $\tau_{5}=3$. This also occurs when comparing only the bright penumbral filaments to the quiet Sun: at $\tau_{5}=1$, the average quiet Sun is about $(257 \pm 95) \mathrm{K}$ hotter than the average over the bright filaments, while this difference increases to $(490 \pm 113) \mathrm{K}$ at $\tau_{5}=3$. This feature is not only seen in the average temperatures, but also in the individual temperatures inferred at each pixel. Figure 2 shows the histograms of the difference between the mean quiet-Sun temperature at $\tau_{5}=3$ and $\tau_{5}=1$, minus the same difference at each pixel of the penumbra (red) or at each pixel of the bright filaments (blue). Hereafter, this quantity is referred to as $\xi(T)$,

$$
\begin{aligned}
\xi(T)= & \left\{\overline{T_{\mathrm{qs}}}\left(\tau_{5}=3\right)-\overline{T_{\mathrm{qs}}}\left(\tau_{5}=1\right)\right\} \\
& \left.-\left\{T\left(\tau_{5}=3\right)\right\}-T\left(\tau_{5}=1\right)\right\} .
\end{aligned}
$$

Locations where $\xi(T)<0$ are regions where the temperature stratification $T\left(\tau_{5}\right)$ converges to the mean quiet-Sun stratification as we probe below the visible surface $\left(\tau_{5} \in[1,3]\right.$ or about $70-80 \mathrm{~km}$ below the surface), while pixels where $\xi(T)>0$ correspond to regions where $T\left(\tau_{5}\right)$ diverges from the mean quietSun stratification. The determination of the temperature in this region has been possible because the analyzed spectral lines (i.e., three Fe I lines located at $1565 \mathrm{~nm}$ ) convey reliable information

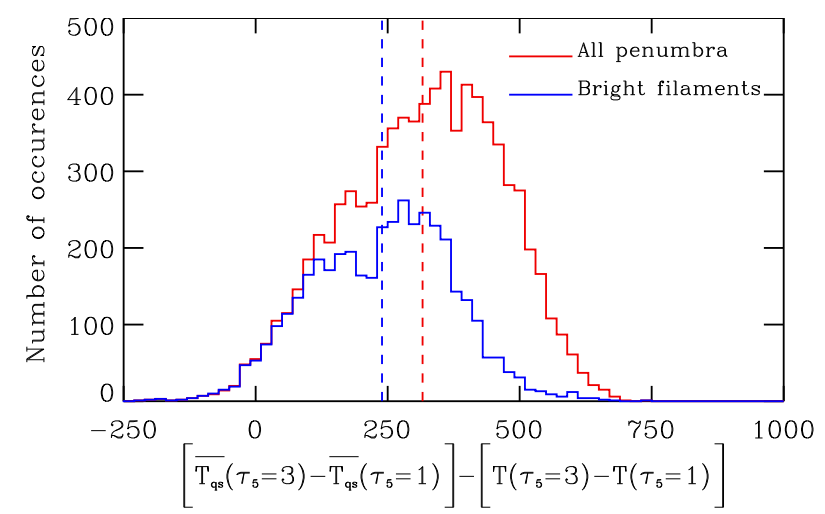

Fig. 2. Histograms of $\xi(T)$ as defined in Eq. (1). Red lines correspond to the penumbral region (bottom panels in Fig. 1). Blue lines correspond to the bright penumbral filaments (white contours in the bottom panels of Fig. 1). The vertical dashed lines indicate the mean values of the histograms, $\overline{\xi(T)}$.

about the temperature at this depth. A similar study employing data from Hinode/SP is not possible because of the large uncertainties in the retrieval of the temperature at $\tau_{5}=3$ using the Fe I spectral lines at $630 \mathrm{~nm}$.

Most of the penumbra and bright filaments are formed by pixels where $\xi(T)>0$, with pixels where $\xi(T)<0$ representing only $1.3 \%$ and $2.8 \%$ of the total, respectively. As in Borrero et al. (2016), we have checked that the results we present here are independent of the amount of wide-angle scattered light considered during the deconvolution process: the very same results are obtained when considering $40 \%$ of the wide-angle scattered light as well as when analyzing the original (i.e., not deconvolved) Stokes vector.

The vertical dashed lines in Fig. 2 indicate the mean value of the histograms. We refer to it as $\overline{\xi(T)}$. They are located at at $\overline{\xi(T)}=(327 \pm 147) \mathrm{K}$ for the entire penumbra (dashed red) and at $\overline{\xi(T)}=(233 \pm 147) \mathrm{K}$ for the bright penumbral filaments (dashed-blue).

We have found that both on average and in $98.7 \%$ of the studied penumbral area, $\xi(T)>0$, which implies that the temperature gradient below the visible surface of the penumbra is lower than the mean temperature gradient of the quiet Sun. A particular interpretation that can be drawn from these results is related to the penumbral thickness below the visible surface. The concepts of thin and thick penumbrae have been discussed on theoretical grounds (see, e.g., Jahn \& Schmidt 1994; Solanki 2003), but so far it was not possible to distinguish them observationally. While in a thin penumbra the magnetopause (defined as the current sheet separating the penumbral magnetic field from the surrounding convection) is located at $\tau_{5} \approx 1$, in a thick penumbra it lies at greater depth. Therefore, if the penumbra were thin, the temperature between $\tau_{5} \in[1,3]$ would converge toward quiet Sun values, and therefore feature $\xi(T)<0$. However, our results show that the opposite occurs: $\xi(T)>0$, thereby favoring a thick penumbra.

It has also been suggested that while in the penumbral spines (regions of stronger and less strongly inclined magnetic field) the magnetic field reaches farther down, in the penumbral intraspines (regions of weaker and more inclined magnetic field) the magnetic field quickly yields to field-free convection below $\tau_{5}=1$ (see Fig. 3 in Spruit \& Scharmer 2006). Interestingly, this latter possibility can be ruled out because the high spatial resolution $\left(\approx 0.4-0.45^{\prime \prime}\right)$ spectropolarimetric data of GREGOR 
allow us to establish that $\xi(T)>0$ also below bright penumbral filaments (i.e., intraspines), both on average and in $97.2 \%$ of the area covered by bright filaments.

\subsection{Bright filaments vs. granules}

Notwithstanding the previous results about the penumbral thickness, there is compelling evidence for the existence of convective-like motions inside the magnetized bright penumbral filaments (Zakharov et al. 2008; Rempel 2012; Tiwari et al. 2013), in particular of some form of convective upflow at their center. In the optically thick region we are studying $\left(\tau_{5} \geq 1\right)$, the thermal stratification $T\left(\tau_{5}\right)$ is dominated by the adiabatic expansion (i.e., cooling) of the plasma as it rises. Because of this, we might surmise that the temperature gradient in the quiet-Sun granules and bright penumbral filaments would be very similar. Our results indicate, however, that the opposite occurs (see Table 1). A possible solution to this seemingly contradictory results can be offered by considering that while in the quiet Sun this adiabatic expansion can take place in all spatial directions, in bright penumbral filaments (i.e., intraspines) it will be limited by the magnetic pressure and tension of the external field (i.e., spines). Consequently, the expansion of the magnetized upflow inside the bright filament will have fewer degrees of freedom, thereby leading to a decreased cooling and thus the smaller temperature gradient observed.

Acknowledgements. The 1.5-m GREGOR solar telescope was built by a German consortium under the leadership of the Kiepenheuer-Institut für Sonnenphysik in Freiburg with the Leibniz-Institut für Astrophysik Potsdam, the Institut für Astrophysik Göttingen, and the Max-Planck-Institut für Sonnensystemforschung in Göttingen as partners, and with contributions by the Instituto de Astrofísica de Canarias and the Astronomical Institute of the Academy of Sciences of the Czech Republic. Financial support by the Spanish Ministry of Economy and Competitiveness through projects AYA2014-60476-P and Consolider-Ingenio 2010 CSD2009-00038 are gratefully acknowledged. We would like to thank Matthias Rempel, Fernando Moreno-Insertis, and Oskar Steiner for fruitful discussions. This research has made use of NASA's Astrophysics Data System.

\section{References}

Borrero, J. M., Asensio Ramos, A., Collados, M., et al. 2016, A\&A, 596, A2 Cally, P. S. 2005, MNRAS, 358, 353

Chandrasekhar, S. 1960, Radiative transfer (New York: Dover)

Chandrasekhar, S., \& Breen, F. H. 1946, ApJ, 104, 430

Collados, M. 2007, in Modern solar facilities - advanced solar science, eds. F. Kneer, K. G. Puschmann, \& A. D. Wittmann, 143

Collados, M., López, R., Páez, E., et al. 2012, Astron. Nachr., 333, 872

Couvidat, S., Birch, A. C., \& Kosovichev, A. G. 2006, ApJ, 640, 516

Danielson, R. E. 1961, ApJ, 134, 289

Deinzer, W. 1965, ApJ, 141, 548

del Toro Iniesta, J. C. 2003, Introduction to Spectropolarimetry (Cambridge, UK: Cambridge University Press)

Franz, M., Collados, M., Bethge, C., et al. 2016, A\&A, 596, A4

Gingerich, O., Noyes, R. W., Kalkofen, W., \& Cuny, Y. 1971, Sol. Phys., 18, 347

Gizon, L., Schunker, H., Baldner, C. S., et al. 2009, Space Sci. Rev., 144, 249

Heinemann, T., Nordlund, Å., Scharmer, G. B., \& Spruit, H. C. 2007, ApJ, 669, 1390

Jahn, K., \& Schmidt, H. U. 1994, A\&A, 290, 295

Khomenko, E., \& Cally, P. S. 2012, ApJ, 746, 68

Khomenko, E., \& Collados, M. 2015, Liv. Rev. Sol. Phys., 12, 6

Lagg, A., Solanki, S. K., Doerr, H.-P., et al. 2016, A\&A, 596, A6

Martínez González, M. J., Pastor Yabar, A., Lagg, A., et al. 2016, A\&A, 596, A5

Meyer, F., Schmidt, H. U., Wilson, P. R., \& Weiss, N. O. 1974, MNRAS, 169, 35

Moradi, H., Baldner, C., Birch, A. C., et al. 2010, Sol. Phys., 267, 1

Parker, E. N. 1979, ApJ, 234, 333

Rempel, M. 2011, ApJ, 729, 5

Rempel, M. 2012, ApJ, 750, 62

Rempel, M., Schüssler, M., Cameron, R. H., \& Knölker, M. 2009, Science, 325, 171

Ruiz Cobo, B., \& Asensio Ramos, A. 2013, A\&A, 549, L4

Ruiz Cobo, B., \& del Toro Iniesta, J. C. 1992, ApJ, 398, 375

Scharmer, G. B., \& Spruit, H. C. 2006, A\&A, 460, 605

Schlichenmaier, R., Bruls, J. H. M. J., \& Schüssler, M. 1999, A\&A, 349, 961

Schmidt, W., von der Lühe, O., Volkmer, R., et al. 2012, Astron. Nachr., 333, 796

Schunker, H., \& Cally, P. S. 2006, MNRAS, 372, 551

Solanki, S. K. 2003, A\&ARv, 11, 153

Spruit, H. C., \& Scharmer, G. B. 2006, A\&A, 447, 343

Tiwari, S. K., van Noort, M., Lagg, A., \& Solanki, S. K. 2013, A\&A, 557, A25

Zakharov, V., Hirzberger, J., Riethmüller, T. L., Solanki, S. K., \& Kobel, P. 2008, A\&A, 488, L17 